\title{
REPETITIVE FEATURE EXTRACTION WITH LOCALIZED DELAUNAY TRIANGULATION FOR COPY-MOVE- FORGERY-DETECTION
}

\author{
Dhanya R, \\ Research Scholar, Department of Computer Applications, \\ Noorul Islam Centre for Higher Education., Thuckalay, Kanyakumari District, TamilNadu, India \\ dhanyamanoj2003@gmail.com \\ R Kalaiselvi \\ Associate Professor, Department of Computer Science and Engineering, \\ Noorul Islam Centre for Higher Education, Thuckalay, Kanyakumari District, TamilNadu, India \\ kalaiselvir32@gmail.com
}

\begin{abstract}
:
The newly invented sophisticated softwares like Adobe Photoshop make the alteration of image very easy. Advanced tools are provided in these softwares that make the image look real which makes it inconvenient to be identified by the naked eye. Taking out features with key point based method using Scale Invariant Feature Transform (SIFT) is discussed in this paper. The extracted feature points are then modeled to with Delaunay Triangulation to get a group of triangles. Mean vertex descriptor of these triangles is matched. Outlier detection is done using Random Sample Consensus (RANSAC). Equivalent methods are outdone by the proposed approach in implementation.
\end{abstract}

Keywords: Forensic Digital Image Processing; Delaunay Triangulation; Forgery Detection; RANSAC; SIFT.

\section{Introduction}

The present state shows the significance of digital images in human life. The editing programmes available now have made the tampering of pictures a child's game. Even before law, this made the images trustless. Image forensic thus ascertains the importance of tools for forensics to distinguish real image from the false one. Some type of forgery is detected by some of the existing methods, but the practical situation shows decrease in accuracy.

Forensic digital image processing mainly uses two approaches [Ng and Chang, (2004)], Active methods and Passive methods. Embedding additional information about the source in the image itself forms active method while the contents are analyzed to see whether the image is altered forms the Passive method [Farid, (2009)].

Copy move forgery is one passive technique where an image portion is copied and replicated in same image itself, but in another portion. The purpose of this forms covering something or overemphasizing it. Purposefully done tampering is not easily identified by the detecting softwares. This is because, usually the irregularities are oppressed of the statistical features locally of various regions in the picture and in the case of passive method; the copied region will show consistency with the other regions. In that case there is a possibility of identifying original areas as forged areas. Thus a filtering method is needed to filter out the outliers. The geometrical matches used for this purposes suffer from high computational costs. The increase in the number of false matches leads to degraded performance and this also happens if the image is having highly consistent regions. For tackling this problem, many of the experiments consider these regions omitted. The size of the image also matters when the image is having more even parts.

A new method is proposed in this paper in which considering the uniqueness metric, the image is enclosed adaptively. The concentration of the key points is also taken into consideration keeping in mind the suspected tampered regions. By using Delaunay Triangulation method, triangles are formed from these points. Then improvement is done repeatedly to create more pixel based accuracy. Suspected regions are more concentrated over the iterations and thus it results in low computational cost. In terms of accuracy and computational cost, the proposed method shows merit. 
The article is structured as follows. Section II covers the extensive literature review conducted on the former methods of CMFD. The proposed method is discussed in Section III and the analysis of the proposed method with experimental support is made in Section IV.

\section{Literature survey}

A number of different methods have been recommended for identifying copy move forgery. There are two methods, namely; key point and block based. Feature elements are mined from the image in both the cases and local feature similarity is evaluated. Feature point is taken out from each point in block based method and in the meantime, key point descriptor is taken as element of interest in the other method.

Framework general for CMFD is illustrated in Fig.1. A variant was presented by [Christlein et al.(2010)]. The first stage aim is to improve the efficiency. The advantage of segmentation is the reduced computational complexity. In the localization phase, local patch centers are determined. From each segment, feature vector is found out. Similar blocks are identified in the matching phase. Filtering phase avoids the spurious pairs. Morphological operations are employed in the post - processing phase. It is done in an iterative manner and the facts from the previous iterations will update the matching information.

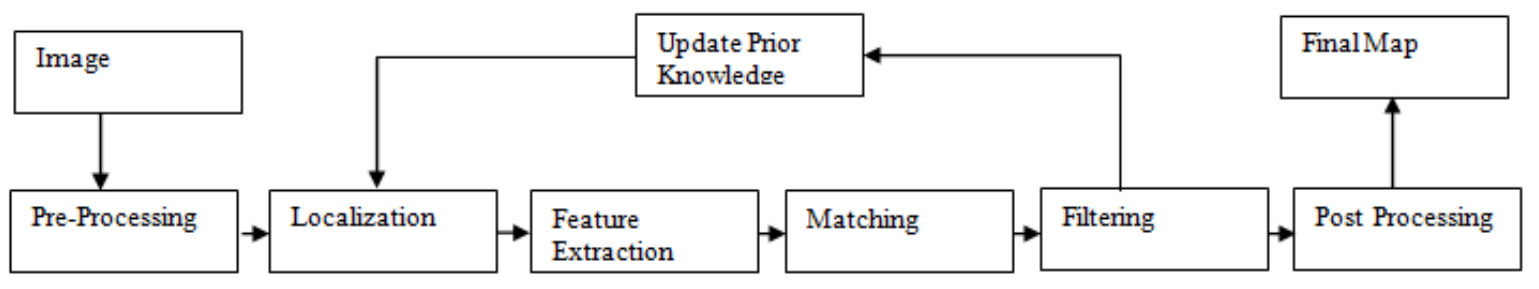

Fig. 1. CMFD - General Framework

The study on CMFD was commenced in 2003[Fridrich et al.,(2003)] by extracting coefficients of DCT from overlapping regular blocks. SVD [Gouhui et al.,(2007)] and PCA [Bashar et al.,(2010)] were used, and were established as vital towards blurring, JPEG compression and noise addition. In the case of images with geometric translations, rotation invariant features like Fourier Mellin Transform (FMT) [Beyram et al.(2009)] and some researchers made use of Local Binary Patterns (LBP) [Leida and Zhu, (2013)]. Polar coordinates represented using circular blocks were used in Polar Cosine Transforms [Li, (2013)] and then in Zernike moments [Ryu et al.(2013)]. The filtering algorithm RANSAC estimates the affine transformations of the duplicated areas [Lowe,(2004)]. For each block, Matching threshold is used.

Feature extraction techniques are mostly used by sparsely sampled methods. SIFT (Scale Invariant Feature Transform) [Amerini et al.(2011), Xunyu et al.(2010)] and SURF (Speeded Up Robust Features) [Bay et al., (2005), Shivakumar abd Baboo,(2011)], are used to identify the prominent points. Due to the decreased number of points analyzed, these methods show very less computational cost and are found to be tough over geometric conversions. Each application has its own limitations and the indicators suitable for one will not be good for another. The interest points are concentrated on large information parts and as a consequence, regions like low contrast areas, where interest points are disproportionately isolated are avoided. Most recently a new method based on Expectation-Maximization method [Li et al., (2014)] was used to minimize the affine transformation errors, which also suffered from high computation cost when the image is very large.

A new method utilizing the advantages of Block-based and Key-point based methods is proposed here in the next section. The number of points which are scrutinized is compared to be very less even if the image is fully covered. The result is low computational cost and the entire procedure concentrates clearly on the mistrusted area during the subsequent repetitions that provides added accurate value.

\section{Proposed method}

Adaptive full coverage of the image including the less contrast region is applied in the proposed method. SIFT is used to detect the interest points. An operative filtering algorithm is used to filter out false positives. Information previously collected is used repeatedly to improve the outcomes.

\subsection{Feature extraction}

The self-similarity of images will be measured as patches particularly in low entropy regions. The usage of all possible spots will decrease the chance of recognition. In large areas with less uniqueness, the likelihood of mock equivalent increases the discernment [Zandi et al., (2016)]. This has led to the selection of more individual points which are less probable to be unequal. But on the contrary, the result will be more accurate if we are to 
include more points of interest in the entire image. The proposed algorithm will take into consideration this situation which adjusts the concentration of interest points grounded on the resident uniqueness. With the help of extensive experiments, after considering the adjacent blocks, the researchers have recognized the exceptionality metric [Barnes et al., (2009)]. They also put forward a local capacity, on the basis of the certainty level and individuality to polish up the solidity of the points of interest. In the first round, the certainty level is set as equal. Image resolution increased will limit the resident differences. And as a result, the preliminary certainty level value relies on resolution of the image. In the subsequent iterations, this prior information will provide the required data.

Primarily additional special points are chosen. For larger dimensions an interest point is taken into consideration when it is greater than the local density. Gaussian window is utilized in finding the local density of each individual point. The smaller value of the distance coefficients will contribute to the nearby point's sensitiveness and this makes the interest points scatter. Interest points that scatter over the entire image are considered in this projected method. The forged areas will not be identical and moreover will be distorted when equated with the original image.

In CMFD, more accurate detection is shown by Polar Cosine Transform (PCT) and Zernike moments. Various filtering techniques are there in which alterations are assessed. RANSAC (Random Sample Consensus was employed in rejecting the outliers or uneven points during affine transformations. In [Barnes et al.,(2011)], PatchMatch is recommended and for every block, adaptive similar threshold is used. PCT is used for its maximal discrimination property [Pew et al.,(2010)].

The PCT features of a continuous image $\mathrm{k}$ with order $\mathrm{m}$ repeated an $\mathrm{n}$ time is calculated as:

$$
f=\left\{\left|M_{i, n}\right| \text { such that } m+1 \leq 3.0 \leq m, n<3 .\right.
$$

where,

$$
M_{i, n}=\Omega_{m} \int_{0}^{2 \pi} \int_{0}^{1}\left[H_{m, n}(r, \theta)\right] k(r, \theta) r d r d \theta .
$$

The interest point selection is done on the basis of a square block surrounding each interest point changed to feature region. The phase of PCT is used in finding the rotation angle among the patch pair.

A method, SIFT (Scale Invariant Feature Transform) is experimentally estimated as invariant to noise, resizing, lighting adjustments, rotations, Gaussian noises and JPEG compression by Huang et al.[Huang et al., (2008)]. Characterization of the extracted features from the area is done using group of linked triangles. Delaunay triangulation, a prototypical mainly applied in computer graphics, is used to relate the feature points extracted from the image. Thus characterization is done by triangles contended with identical feature pixels. This method is a variation of Voronoi tessellation whose drawback is that the minutest element omits the edges and the contents considered to be consistent. Mean vertex of these triangles is used for the matching purposes.

\subsection{Matching of Triangular points}

From the geometric vertices, Mean Vertex of each triangle is obtained. It is done by taking the average value of the feature vectors given as Vavg $=(\mathrm{V} 1 \mathrm{i}+\mathrm{V} 2 \mathrm{i}+\mathrm{V} 3 \mathrm{i}) / 3$ where $\mathrm{i}$ shows the extracted feature vectors ranging from 1 to the number of triangles formed through Delaunay triangulation in the entire image. On account of the Li norm of the Mean Vertex, the triangles obtained are sorted. This helps in finding the possible forged regions. MVs thus obtained from every triangle are matched with one another and with a fixed length window in place of an adaptive window. Matches are considered only when the relative displacement of $\mathrm{Li}$ with their corresponding MV shows a decreased threshold value. Equating two triangles with $\mathrm{x}$ and $\mathrm{y}$ being the triangle pair indices, and given $\operatorname{Vmx}$ and $V m y$ as the equivalent Mean Vertex, then $|\operatorname{Vmx}-\operatorname{Vmy}| \leq \mathrm{THv}(\mathrm{y}-\mathrm{x})<$ ws, where ws shows the window size and Thv, the threshold value.

\subsection{Identifying False Positives}

Because of the inherent self-resemblance, the real images show common pseudo assignment of the suspected matched pairs. The areas formed from the same copy paste action shows similar behavior and this helps in eliminating the pseudo matches. Affine transforms are used here to compute the relationship common to both. Most of the filtering algorithms suffer from high computational cost. RANSAC (Random Sample Consensus) handles this problem very effectively. In spite correct matches are incorrectly represented as false pairs. Points of interest found out are denser than the key points. The grouping of these points, that augments the likelihood of finding out correct pairs, is not done as it produces high computational complexity. 


\section{Experimental setup}

Experimental analysis of the system proposed is done in this section and it is matched against the most updated CMFD methods. MATLAB 2017a is used for Experimental analysis with $2 \mathrm{GHz}$ Intel Core i3-5005U CPU using 64-bit Operating System and 4 GB RAM. The evaluation is done with diverse subsets of traditional datasets. These datasets include modest copy move to images with transformations like rotation, compression, resizing, multiple forgery and combined transformations.

\subsection{Datasets used}

For analysing the comparative performance of the proposed method with that of the prevailing methods, we have used MICC-F220 [14], that includes 110 true images along with 110 altered images with different transformations.

\subsection{Metrics}

Pixel level evaluation of the images is done with the following metrics.

\begin{tabular}{|l|l|l|l|}
\hline SI No & \multicolumn{1}{|c|}{ Parameter Name } & \multicolumn{1}{|c|}{ Formulae } & \multicolumn{1}{c|}{ Description } \\
\hline 1 & & & $\begin{array}{l}\text { TP }- \text { Truepositive or Forged } \\
\text { pixels identified as Forged } \\
\text { pixely on Tampered image } \\
\text { FN - FalseNegative or } \\
\text { Forgedpixels not identified as } \\
\text { forged pixels on Tampered } \\
\text { image }\end{array}$ \\
\hline 1 & TruePositiveRate & $\begin{array}{l}\text { TPR }=\mathrm{TP} / \\
(\mathrm{TP}+\mathrm{FN})\end{array}$ & $\begin{array}{l}\text { TN }- \text { TrueNegative or Original } \\
\text { pixels identified as original } \\
\text { pixels on Tampered image } \\
\text { FP }- \text { False Positive or Original } \\
\text { pixels identified as Forged } \\
\text { pixels on Tampered image }\end{array}$ \\
\hline 3 & FrueNegativeRate & $\begin{array}{l}\text { RNR }=\mathrm{TN} / \\
(\mathrm{TN}+\mathrm{FP})\end{array}$ & $\begin{array}{l}\text { Represent rate of true matching } \\
\text { or correct output }\end{array}$ \\
\hline 4 & FalseNegativeRate & $\begin{array}{l}\text { FPR }=\mathrm{FP} / \\
(\mathrm{FP}+\mathrm{TP})\end{array}$ & $\begin{array}{l}\text { Represent rate of false matching } \\
\text { or wrong output }\end{array}$ \\
\hline 5 & Accuracy & $\begin{array}{l}(\mathrm{TP}+\mathrm{FN}) / \mathrm{TN}) \\
(\mathrm{TN}+\mathrm{FP}+\mathrm{TP}+\mathrm{FN})\end{array}$ & Accuracy in numeric form \\
\hline
\end{tabular}

Table 1. Pixel level evaluation metrics

Precision : - Number of appropriately identified tampered pixels in the entire figure of tampered

Pixels given as TPR / (TPR + FPR)

Recall : - Ratio of true positives to the predicted results

F1 - Score : - Harmonic mean of the precision and recall limiting between 0 and 1

$$
\text { F1 Score }=2 \times \frac{P x R}{P+R}
$$

\section{Results and discussions}

Experimental analysis of the proposed technique with existing method done in this section shows that more localization happens in this approach and it has enhanced the accuracy. F1 score calculated shows this. The recall rate is enhanced as a result of the iterative approach and this is the result of considering each and every copied region. This method of hierarchical search also reduced the execution time.

(a)
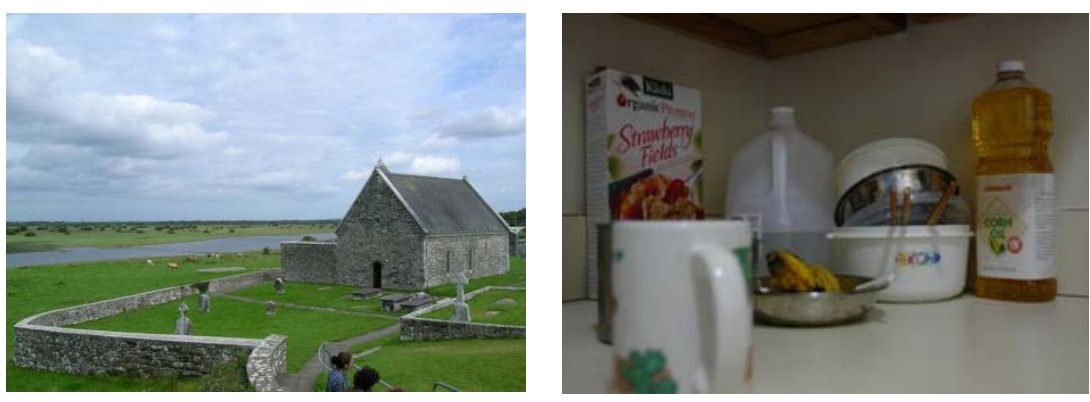
( b)
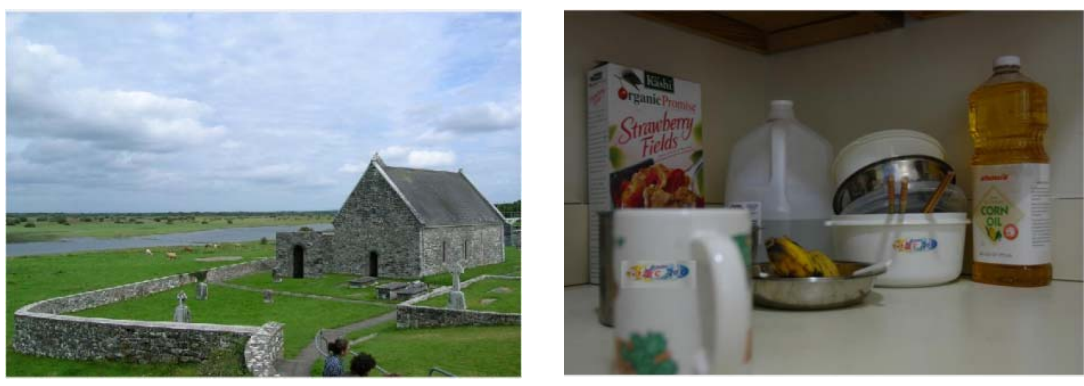

(c)
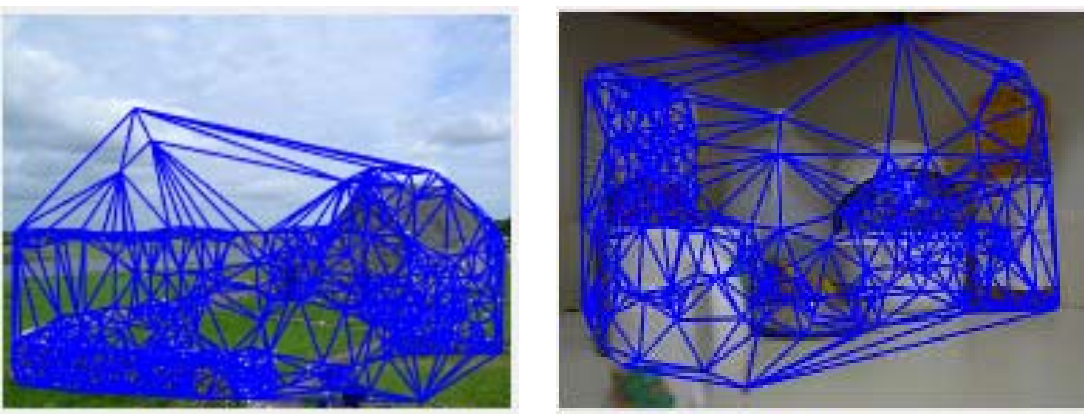

(d)
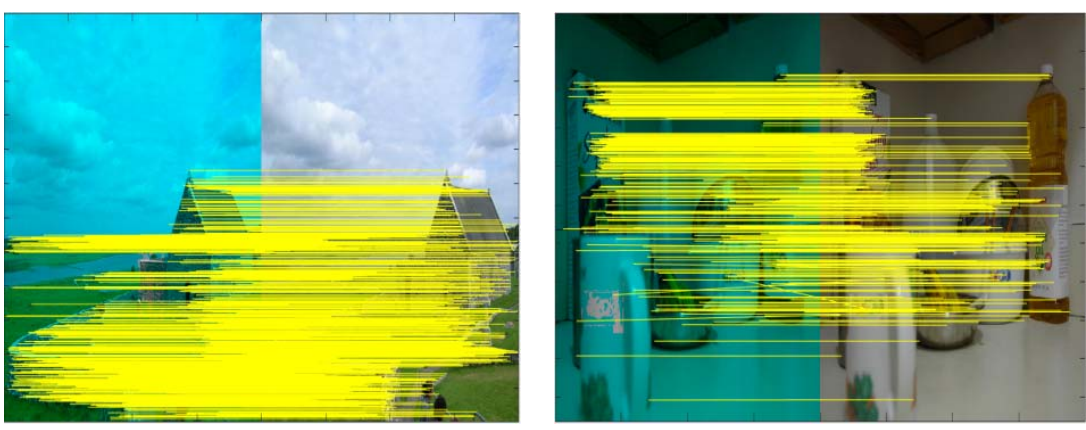

(e )
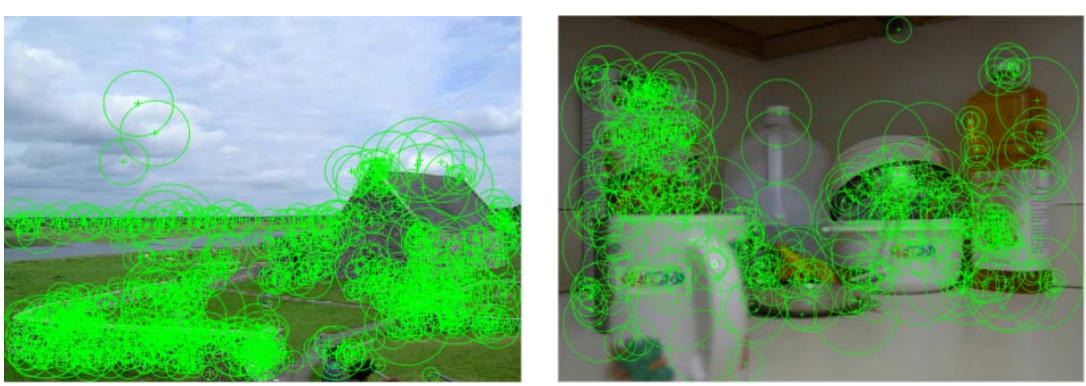

(f)
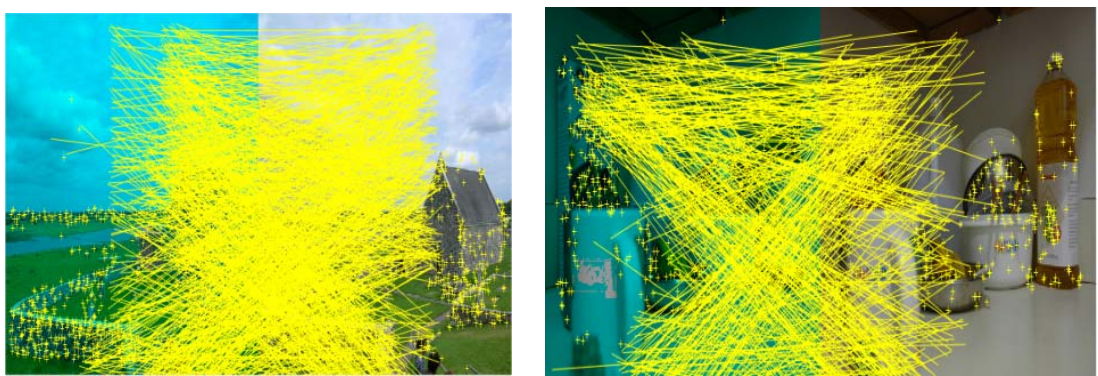
(g)
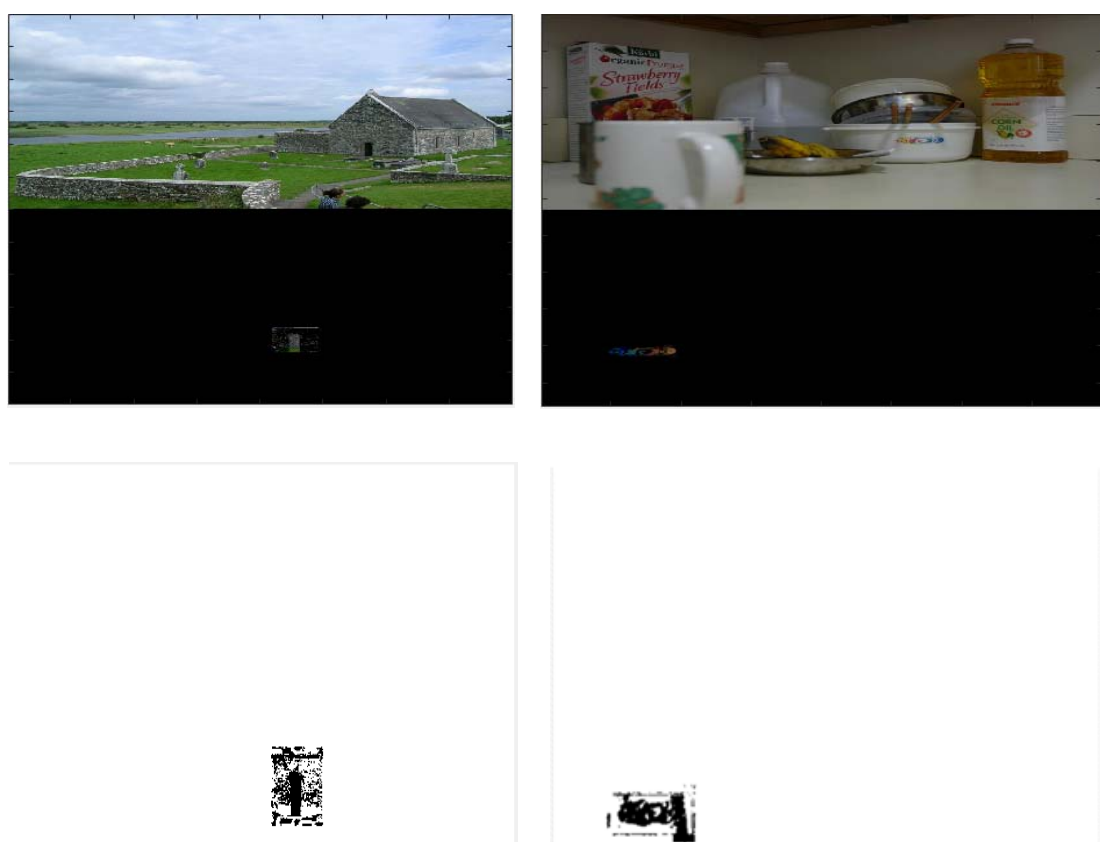

(h)

Fig 2 - Implementation result - a) Real Image, b) Tampered image c) Delaunay Triangulation d) SIFT matched features e) SURF matched features f) Delaunay matched features g) Tampered region detection

h) Binary image

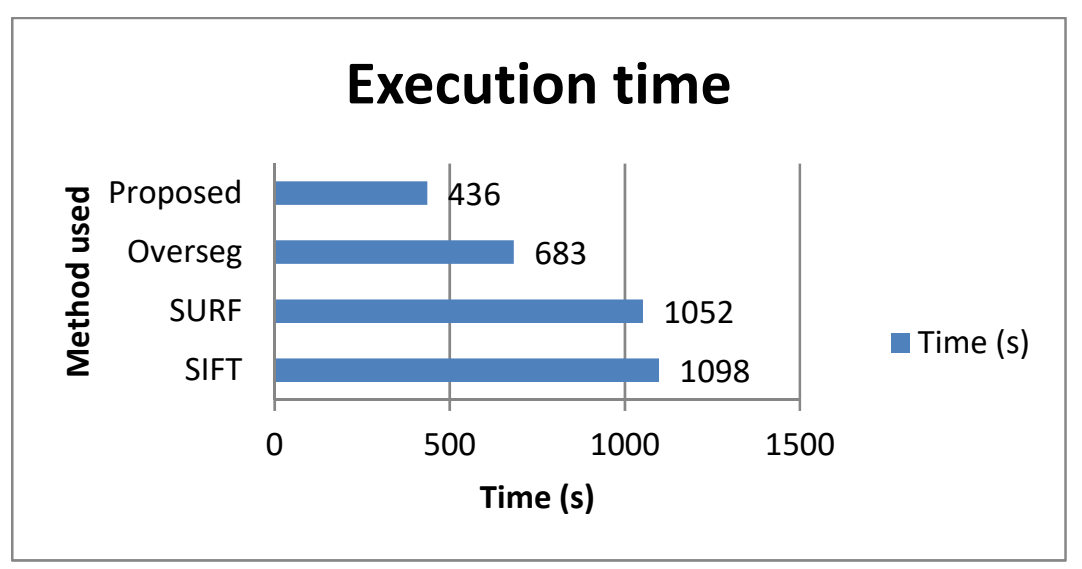

Fig 3: Execution time Comparison

\begin{tabular}{|l|c|c|}
\hline Method & Precision & Recall \\
\hline SIFT & 0.69 & 0.91 \\
\hline SURF & 0.95 & 0.63 \\
\hline Proposed & 0.99 & 0.89 \\
\hline
\end{tabular}

Fig 4: Precision and Recall 


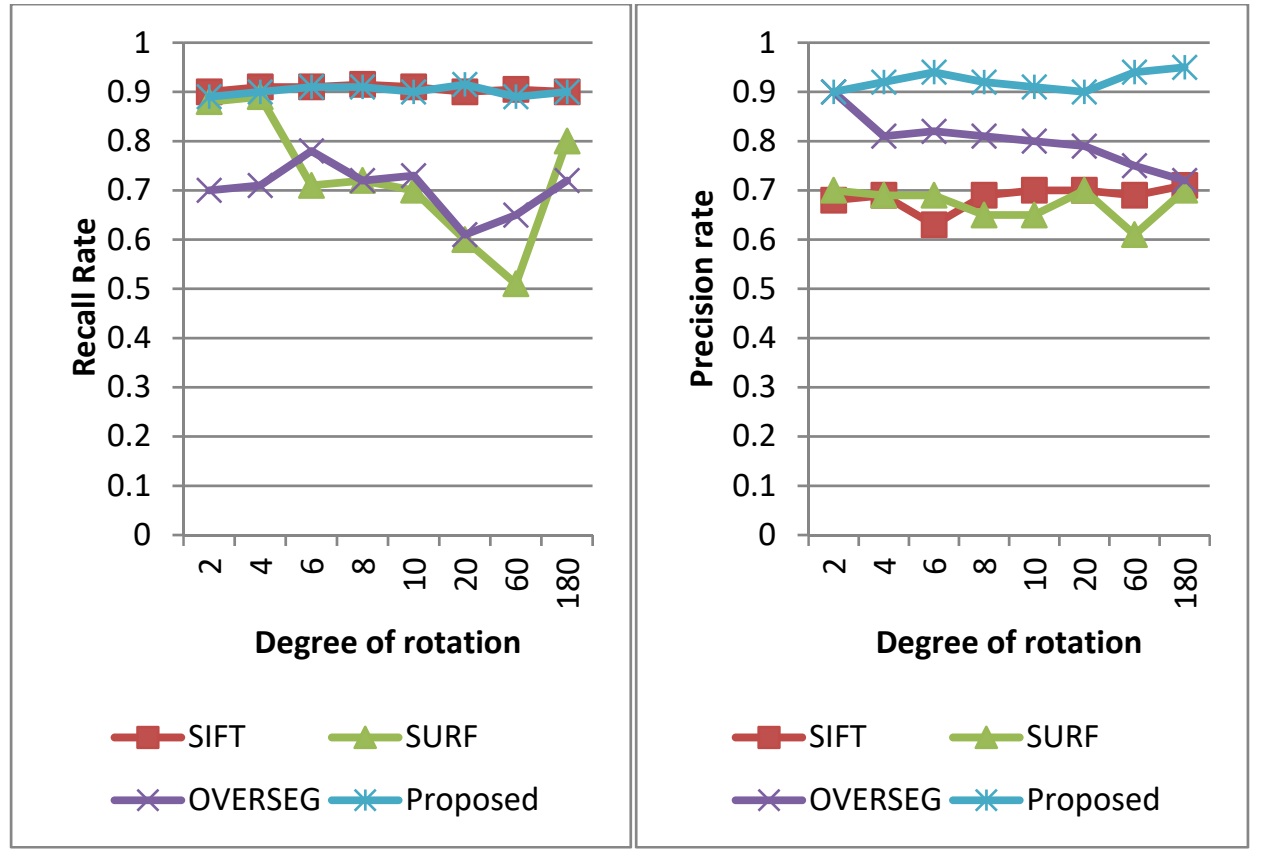

Fig 5: Rotation : - Recall

Precision

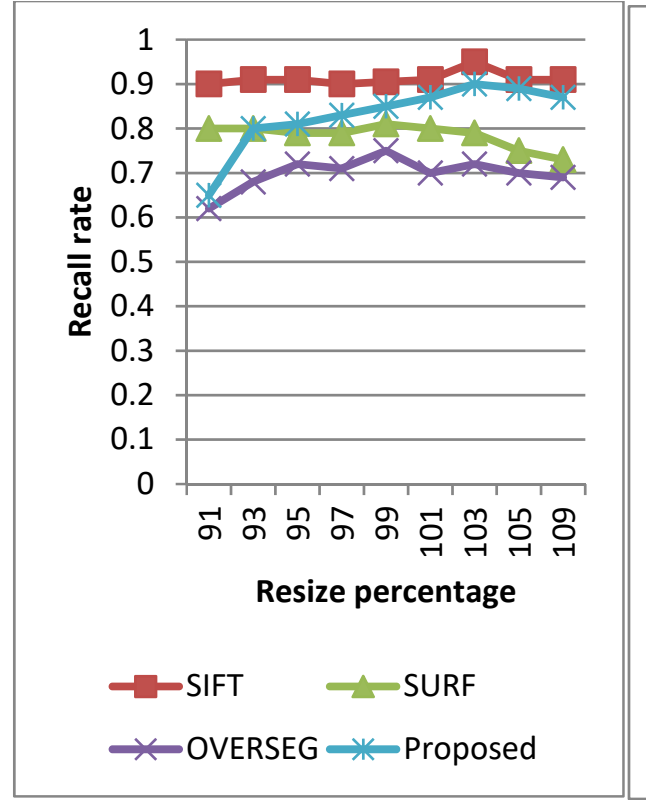

Fig 6: Resizing - Recall

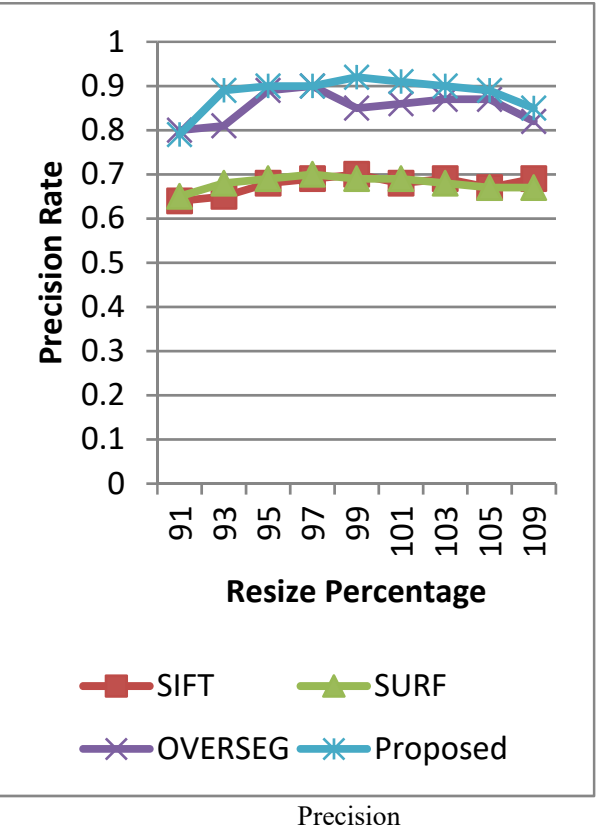

\section{Conclusion}

A different approach is experimented in this paper. The points of interest present per area are very large and the possibility of identifying the tampered region very sharply will be effectively increased for the unique areas. Iterative approach is used to focus largely on the significant regions of tampering. This is done with the data poised from the earlier iterations. The technique projected will effectively select the samples taking into consideration under sampled and oversampled. Experimental analysis shows that the method proposed is far better in case of many affine transformations like scaling, rotation, and multiple pastes. More concentration will be put on the JPEG compression in the forthcoming work.

\section{Acknowledgements}

No funding sources

\section{References}

[1] Amerini,I; Ballan,L; Caldelli, L; DelBimbo; A and Serra,G.(2011): A SIFT based forensic method for copy-move attack detection and transformation recovery, IEEE Transaction of Information Forensics Security, Vol.6, pp. 1099-1110. 
[2] Barnes, C; Goldman,D.B; Scechtman, E and Finkelstein A.(2009): The PatchMatch: a randomized correspondence algorithm for structural image editing, ACM Trans. Graph, Vol. 28, pp. 1-11.

[3] Barnes,C; Goldman,D.B; Scechtman, E and Finkelstein, A.(2011): The PatchMatch randomized matching algorithm for image manipulation," Communication ACM, Vol.54, pp.103-110.

[4] Bashar,M; Noda, K; Ohnishi, N and Mori, K.(2010): Exploring duplicated regions in natural images, IEEE Transactions in Image Processing, pp. 1-1.

[5] Bay,H; Ess,A; Tuytelaars, T and Van Gool,L.(2008): Speeded up Robust features (SURF), Computer Vision and Image Understanding, Vol. 110, pp. 346-359.

[6] Beyram,S; Sencar, H T and Memon, N. (2009): An Efficient and robust method for detecting copy move forgery, IEEE ICASSP, pp. $1053-1056$.

[7] Christlein, V; Riess C, Jordan J and Angelopoulou, E.(2010): An evaluation of popular copy-move forgery detection, IEEE In. Workshop on Information Forensics and Security, pp, 1-6.

[8] Farid, H.(2009): Image Forgery detection, IEEE Signal Process Magazine, Vol.26, pp.16-25.

[9] Fridrich, J; Soukalm,D; Lukáš, J. (2003): Detection of copy-move forgery in digital images, In proceedings of the Digital Forensic Research Workshop, Cleveland, pp. 19-23.

[10] Guohui,L; Qiong, W; Tu, D and Shaojie,S.(2007): A Sorted neighbourhood approach for detecting duplicated regions in image forensics based on DWT and SVD, IEEE International Conference on Multimedia and Expo, pp.1750-1753.

[11] Huang, H; Guo, W and Zhang, Y. (2008): Detection of Copy-Move forgery in digital Images using SIFT algorithm, Pacific-Asia Workshop in Computational Intelligence and Industrial Application (PACIIA), Computer Society, pp. 272-276.

[12] Leida,S.L; Li Hancheng Zhu.(2013): An Efficient scheme for detecting copy move forged images by local binary patterns, Journal of Information Hiding and Multimedia Signal process., Vol 4, pp,46-56.

[13] Li, J; Li, X, Yang, B and Sun,X.(2014): Segmentation based Image copy-move forgery detection scheme, IEEE Transactions on Information Forensics and Security, Vol.5.

[14] Li,Y.(2013): Image copy-move forgery detection based on polar cosine transform and approximate nearest neighbor searching, Forensic Science Int., Vol 224, pp.59-67.

[15] Lowe, D.G. (2004): Distinctive image features from scale invariant keypoints, International Journal of Computer Vision, Vol.60,pp.91110.

[16] Ng, T. T and Chang, S F. (2004): A model for Image splicing, Proceedings of ICIP, Vol 2. pp. 1169-1171.

[17] Pew-Thian Y; Xudong J and Kot. A. C. (2010): Two dimensional polar harmonic transforms for invariant image representation, IEEE Transactions on Pattern Analysis and Machine Intelligence, Vol. 32, pp.1259-1270.

[18] Ryu, S J; Lee, M.J and Lee,H.K.(2013): Rotation invariant localization of duplicated image regions based on zernike moments, IEEE Transactions on Information Forensics Security, Vol.8,pp.1355-1370.

[19] Shivakumar, B and Baboo S.S. (2011): Detection of region duplication forgery in digital images using SURF, International Journal of Computer Science Issues, Vol.8.

[20] Xunyu, P and Siwei, L.(2010): Detecting image region duplication using SIFT features, IEEE ICASSP, pp. 1706-1709

[21] Zandi, M.; Mahmoudi-Aznaveh, A and Talebpour, A. (2016): Iterative Copy-Move Forgery Detection Based on a New Interest Point Detector. IEEE Transactions on Information Forensics and Security, 11(11),2499-2512. doi:10.1109/tifs.2016.2585118.

\section{Authors Profile}

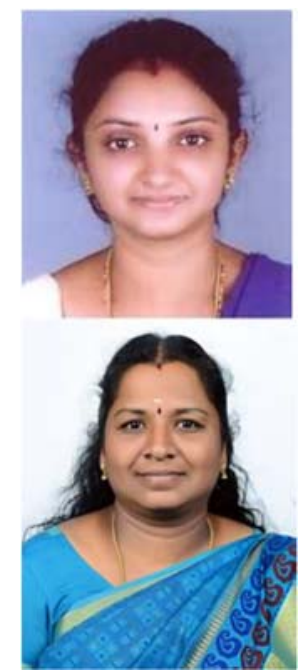

Dhanya R, is a Research Scholar in the Department of Computer Applications at Noorul Islam Centre for Higher Education, Kumaracoil, Thuckalay, Tamil Nadu. She is currently working as an Assistant Professor in the Department of Computer Applications, St. Teresa's College, Ernakulam, Kerala. She is an MCA graduate with research interest in Digital Image Processing and Digital Electronics and with 14 years of teaching experience. She has presented and published papers in International Conferences and Journals.

R. Kalaiselvi, is currently working as an Associate Professor in the Department of Computer Science and Engineering, Noorul Islam Centre for Higher Education, Kumaracoil, Thuckalay, Tamilnadu, India. She has more than eighteen years of teaching experience. She obtained her B.E (Electronics and Communication Engineering) from M.S University, Tirunelveli, and her ME (Computer Science and Engineering) from Anna University, Chennai, India.and her Ph.D from Anna University Chennai. Her research interests include Network Security, Cloud Computing and Distributed Computing. She has published many research papers in National/International Conferences and Journals. She has attended several seminars and workshops in the past ten years. She is an IEEE member. 